\title{
Influence of Kinetic and Thermodynamic Factors on the Glass-Forming Ability of Zirconium-Based Bulk Amorphous Alloys
}

\author{
S. Mukherjee, ${ }^{1, *}$ J. Schroers, ${ }^{1,2}$ W. L. Johnson, ${ }^{1}$ and W.-K. Rhim ${ }^{1}$ \\ ${ }^{1}$ Division of Engineering and Applied Science, Mail Code 138-78, California Institute of Technology, \\ Pasadena, California 91125, USA \\ ${ }^{2}$ Liquidmetal Technologies, Lake Forest, California 92360, USA
}

(Received 11 August 2004; published 21 June 2005)

The time-temperature-transformation curves for three zirconium-based bulk amorphous alloys are measured to identify the primary factors influencing their glass-forming ability. The melt viscosity is found to have the most pronounced influence on the glass-forming ability compared to other thermodynamic factors. Surprisingly, it is found that the better glass former has a lower crystal-melt interfacial tension. This contradictory finding is explained by the icosahedral short-range order of the undercooled liquid, which on one hand reduces the interfacial tension, while on the other hand increases its viscosity.

In recent years, several multicomponent alloys have been developed that can be cast in bulk amorphous form with cooling rates as low as $1 \mathrm{~K} / \mathrm{s}$ [1-3]. Attempts have been made to explain the exceptional stability against crystallization in these systems by both kinetic and thermodynamic principles [4-6]. From a kinetic point of view, the dynamic viscosity is an important parameter to describe the time scale for structural rearrangement of the undercooled liquid atoms for the growth of a crystal nucleus. From a thermodynamic point of view, the better glass former is expected to have a lower thermodynamic driving force for crystallization, which is given by the Gibbs free energy difference between the liquid and crystal, $\Delta G$. Particularly, in classical nucleation theory $[7,8]$, the activation barrier for nucleation, $\Delta G^{*}$ is expressed as

$$
\Delta G^{*}=\frac{16 \pi \sigma^{3}}{3 \Delta G^{2}},
$$

where $\sigma$ is the crystal-melt interfacial tension. It is clear from Eq. (1) that lower thermodynamic driving force and higher interfacial tension will lead to greater stability of the undercooled melt against crystallization. While a number of studies address the influence of thermodynamic and kinetic factors on the stability of undercooled liquids, no studies on their relative importance have been carried out to date. Also, there are no investigations dealing with correlations between the kinetic and thermodynamic factors to determine whether they stem from the same underlying property of the undercooled liquid.

In this study, we investigate the influence of $\Delta G$, melt viscosity, and crystal-melt interfacial tension on the glass-forming ability of three zirconium-based bulk amorphous alloys: $\mathrm{Zr}_{41.2} \mathrm{Ti}_{13.8} \mathrm{Cu}_{12.5} \mathrm{Ni}_{10} \mathrm{Be}_{22.5}$ (Vit1), $\mathrm{Zr}_{57} \mathrm{Cu}_{15.4} \mathrm{Ni}_{12.6} \mathrm{Al}_{10} \mathrm{Nb}_{5}$ (Vit106), and $\mathrm{Zr}_{55} \mathrm{Al}_{22.5} \mathrm{Co}_{22.5}$ (ZAC). These three alloys were chosen because of their widely different glass-forming abilities, but otherwise similar properties. The time-temperature-transformation
(TTT) diagrams of these alloys, which give a quantitative measure of their glass-forming ability, were measured using the high-vacuum electrostatic levitation (HVESL) technique to eliminate any heterogeneous nucleation effects. A detailed description of the ESL facility is given elsewhere [9].

The alloys were prepared from high purity starting materials in an arc melter. The glass-transition temperatures, $T_{g}$, and the liquidus temperatures, $T_{L}$, were obtained for the three alloys by heating with a rate of $0.33 \mathrm{~K} \mathrm{~s}^{-1}$ in a differential scanning calorimeter. The $T_{g}$ and $T_{L}$ values for the alloys investigated in this study are summarized in Table I. Samples about $15 \mathrm{mg}$ in weight (spheres $\sim 2 \mathrm{~mm}$ in diameter) were processed in the HVESL. To determine the TTT diagram, isothermal experiments were performed. Therefore, the molten sample was cooled to a predetermined temperature by turning off the laser, which was subsequently turned back on at a preset power to maintain an isothermal temperature.

Isothermal crystallization times are summarized as TTT curves for the three alloys that are shown in Fig. 1. All three TTT curves have the " $\mathrm{C}$ " shape that arises from the competition between increasing thermodynamic driving force for crystallization and decreasing atomic mobility with increasing undercooling. The fits of the TTT curves using classical nucleation theory (CNT) are also shown in

TABLE I. Characteristic temperatures $\left(T_{g}, T_{L}\right.$, and $\left.T_{\text {nose }}\right)$, isothermal time at the nose temperature of TTT curve $\left(\Delta t_{\text {nose }}\right)$, and critical cooling rates $(R c)$.

\begin{tabular}{lccccc}
\hline \hline BMG & $\begin{array}{c}T_{g} \\
(\mathrm{~K})\end{array}$ & $\begin{array}{c}T_{L} \\
(\mathrm{~K})\end{array}$ & $\begin{array}{c}T_{\text {nose }} \\
(\mathrm{K})\end{array}$ & $\begin{array}{c}\Delta t_{\text {nose }} \\
(\mathrm{s})\end{array}$ & $\begin{array}{c}R c \\
\left(\mathrm{~K} \mathrm{~s}^{-1}\right)\end{array}$ \\
\hline Vit1 & 620 & 993 & 800 & 70 & 2 \\
Vit106 & 682 & 1115 & 875 & 6 & 10 \\
ZAC & 753 & 1323 & 980 & 2 & 18 \\
\hline \hline
\end{tabular}




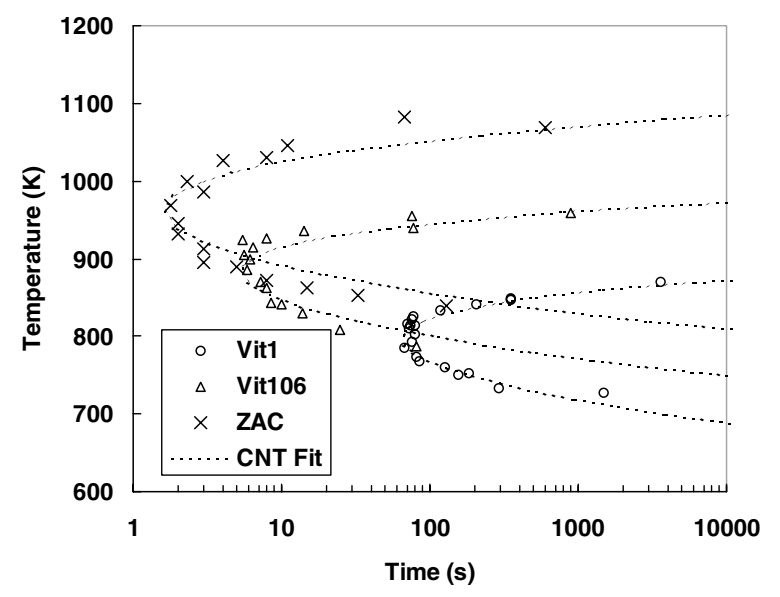

FIG. 1. TTT curves of $\mathrm{Zr}_{41.2} \mathrm{Ti}_{13.8} \mathrm{Cu}_{12.5} \mathrm{Ni}_{10} \mathrm{Be}_{22.5}$ (Vit1), $\mathrm{Zr}_{57} \mathrm{Cu}_{15.4} \mathrm{Ni}_{12.6} \mathrm{Al}_{10} \mathrm{Nb}_{5}$ (Vit106), and $\mathrm{Zr}_{55} \mathrm{Al}_{22.5} \mathrm{Co}_{22.5}$ (ZAC), as well as the classical nucleation theory (CNT) fits.

Fig. 1 and will be discussed later. For Vit1, the temperature at which the time for crystallization is minimum (commonly referred to as the "nose" of the TTT curve) is about $800 \mathrm{~K}$. The corresponding nose time is about $70 \mathrm{~s}$. The nose temperatures $\left(T_{\text {nose }}\right)$, the isothermal times at the nose before crystallization $\left(\Delta t_{\text {nose }}\right)$, and the critical cooling rates $(R c)$ for vitrification are summarized in Table I. The critical cooling rates, as listed in Table I, vary over an order of magnitude illustrating the wide range in glass-forming abilities of these alloys.

The temperature dependent Gibbs free energy difference for Vit1 and Vit106 was determined in earlier publications $[10,11]$. The Gibbs free energy difference for the two alloys was found to be similar at the same relative undercooling level. The entropy of fusion is experimentally found to be almost identical for the three alloys $\left(\Delta S_{f}=\right.$ $8.65,8.5$, and $8.7 \mathrm{~J} / \mathrm{mol} / \mathrm{K}$ for Vit1, Vit106, and ZAC, respectively), and $\Delta G$ for ZAC is estimated to be very similar to Vit1 and Vit106 [12]. Thus, the thermodynamic driving force fails to explain the order of magnitude difference in critical cooling rates of these Zr-based bulk amorphous alloys.

The temperature dependent dynamic viscosities for all three alloys have recently been measured $[13,14]$, and can be described by the Vogel-Fulcher-Tammann (VFT) equation [4,5]: $\eta=\eta_{0} \exp \left[D T_{0} /\left(T-T_{0}\right)\right]$, where $T_{0}$ is re-

TABLE II. Vogel-Fulcher-Tammann viscosity parameters $\left(\eta_{0}\right.$, $\left.D, T_{0}\right)$ and classical nucleation theory TTT fitting parameters $(\alpha$, A).

\begin{tabular}{lccccc}
\hline \hline BMG & $\begin{array}{c}\eta_{0} \\
(\mathrm{~Pa} \mathrm{~s})\end{array}$ & $D$ & $\begin{array}{c}T_{0} \\
(\mathrm{~K})\end{array}$ & $\begin{array}{c}A \\
\left(\mathrm{~m}^{-5}\right)\end{array}$ & $\alpha$ \\
\hline Vit1 & $1 \times 10^{-6}$ & 23.8 & 390.0 & $8 \times 10^{28}$ & 0.35 \\
Vit106 & $15 \times 10^{-6}$ & 11.3 & 524.7 & $7 \times 10^{25}$ & 0.47 \\
ZAC & $6 \times 10^{-6}$ & 12.2 & 576.0 & $5 \times 10^{30}$ & 0.52 \\
\hline \hline
\end{tabular}

ferred to as the VFT temperature, $D$ is called the "fragility parameter," and $\eta_{0}$ is the high temperature limit of viscosity. The viscosity fitting parameters $\left(\eta_{0}, D, T_{0}\right)$ of the three alloys are listed in Table II. To study the influence of viscosity on crystallization time scales, the TTT curves for the three alloys are plotted in Fig. 2 with the temperature axis normalized by their respective $T_{g}$. For clarity, only the fitting curves are shown because there is reasonable agreement between experimental data and the CNT fits. The nose temperatures of the three alloys are at the same position of about 1.3 on the normalized temperature axis. On the secondary $y$ axis of Fig. 2 are shown the dynamic viscosities of the three alloys at the nose temperatures. The viscosities differ by almost 2 orders of magnitude. The almost 2 orders of magnitude difference in the crystallization times of the alloys can be explained by the 2 orders of magnitude difference in their viscosities $\left(t_{x} \sim \eta\right)$. Thus, it is clear from Fig. 2 that dynamic viscosity plays the most decisive role in determining the critical cooling rates of these alloys when other parameters are held constant.

To investigate the role of interfacial tension on crystallization time scales, the TTT curves are analyzed with CNT. According to CNT, the time for crystallization, $t_{x}$, is given by $[8,15]$

$$
t_{x}=\left(\frac{3 x}{\pi I_{\mathrm{SS}} u^{3}}\right)^{1 / 4},
$$

where $x$ is the detectable volume fraction during the starting of the crystallization process (a value of $10^{-3}$ was

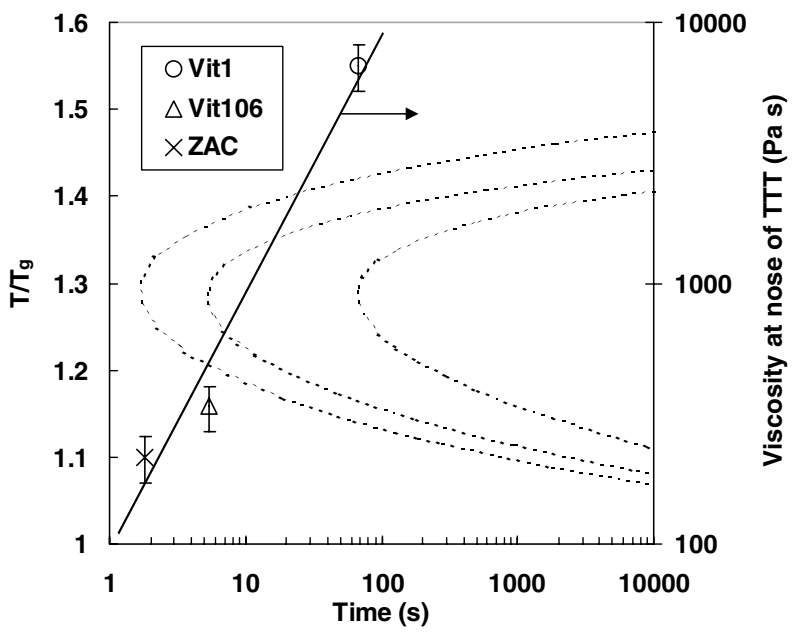

FIG. 2. TTT fitting curves for the three alloys with temperature axis normalized by the glass-transition temperature $\left(T_{g}\right)$. In the order of increasing nose times, the TTT curves correspond to $\mathrm{Zr}_{55} \mathrm{Al}_{22.5} \mathrm{Co}_{22.5}$ (ZAC), $\mathrm{Zr}_{57} \mathrm{Cu}_{15.4} \mathrm{Ni}_{12.6} \mathrm{Al}_{10} \mathrm{Nb}_{5}$ (Vit106), and $\mathrm{Zr}_{41.2} \mathrm{Ti}_{13.8} \mathrm{Cu}_{12.5} \mathrm{Ni}_{10} \mathrm{Be}_{22.5}$ (Vit1). The secondary $y$ axis shows the dynamic viscosities of the three alloys at the nose temperature of the TTT curves. The error bars in the viscosity of $\pm 20 \%$ are shown. The viscosity scales linearly with the crystallization time at the nose temperature. 
used), $I_{\mathrm{SS}}$ is the steady state nucleation rate, and $u$ is the growth rate. Reasonably good fitting is obtained using the above equation for all three alloys as shown in Fig. 1. The steady state nucleation rate is given by

$$
I_{\mathrm{SS}}=A D_{\text {eff }} \exp \left(-\frac{\Delta G^{*} f(\theta)}{k_{B} T}\right),
$$

where $D_{\text {eff }}$ is the effective diffusivity, $A$ is a constant, $k_{B}$ is Boltzmann's constant, $\Delta G^{*}$ is the activation barrier for nucleation [Eq. (1)], and $f(\theta)$ is the catalytic potency factor. Assuming a diffusion controlled mechanism, the growth velocity, $u$, is given by

$$
u=\frac{D_{\text {eff }}}{a}\left[1-\exp \left(-\frac{\Delta G}{k_{B} T}\right)\right],
$$

where $a$ is the interatomic spacing of the alloy. The interatomic spacing is estimated from the measured specific volumes [13,14], and an average value of $2.55 \AA$ is used for all three alloys. The effective diffusivity $\left(D_{\text {eff }}\right)$ was estimated by the Stokes-Einstein equation, $D_{\text {eff }}=$ $k_{B} T /(3 \pi \eta a)$, where $\eta$ is the dynamic viscosity of the liquid. Thus, all quantities are known for the calculation of the steady state nucleation rate [Eq. (3)] and the growth rate [Eq. (4)] except the interfacial tension $(\sigma)$, the constant $A$, and the catalytic potency factor, $f(\theta)$.

To investigate the possible structural effects on the nucleation behavior of phases with different degrees of polytetrahedral order, the negentropic model by Spaepen [16] and Thompson [17] was used. The interfacial tension following such an approach is given by $[16,17]$

$$
\sigma=\alpha \frac{T \Delta S}{\left(N_{A} V_{\mathrm{mol}}^{2}\right)^{1 / 3}},
$$

where $\alpha$ is a factor that depends on the structure difference between the crystal nucleus and the melt, $V_{\text {mol }}$ is the molar volume, $\Delta S$ is the entropy difference between liquid and crystal, and $N_{A}$ is Avogadro's number. In earlier studies $\alpha$ was calculated to be 0.86 for face centered cubic and hexagonal close-packed structures, and 0.71 for a body centered cubic structure [18]. However, $\alpha$ is much smaller $(\sim 0.3)$ for icosahedral quasicrystals $[19,20]$ and increases from 0.3 to 0.7 for phases with decreasing polytetrahedral order.

The entropy difference between the liquid and the crystal decreases with deeper undercooling until it vanishes at the Kauzmann temperature [21]. Microstructural investigations for Zr-based bulk amorphous alloys have revealed a high density of nanocrystals with greater refinement at deeper undercoolings, which suggests a drastic reduction in the nucleation barrier close to the glass-transition temperature [22,23]. A number of mechanisms have been proposed to account for this experimental observation $[24,25]$. To incorporate this effect in CNT, we used temperature dependent entropy $[10,11]$ in Eq. (5), which makes the interfacial tension disappear at the Kauzmann temperature. The TTT curves were fitted using $\alpha$ and $A$ as the only variable parameters (listed in Table II), while $f(\theta)$ is assumed to be one. The validity of assuming homogeneous nucleation $[f(\theta)=1]$ for thermally fluxed, containerless processed samples is discussed in an earlier publication [26]. Vit1 has an $\alpha$ value of 0.35, indicating that the Vit1 melt would form icosahedral quasicrystals, which was, in fact, found experimentally by Waniuk et al. [27]. The $\alpha$ values for the other two alloys suggest the formation of intermediate phases with lower polytetrahedral order $(\alpha=0.47$ and 0.52 for Vit106 and ZAC, respectively) compared to Vit1.

The low values of $\alpha$ in glass-forming alloys compared to pure metals and the nucleation of icosahedral quasicrystals indicates a high degree of polytetrahedral order in the undercooled liquid. Recent in situ experiments [28] provide direct evidence of icosahedral short-range order (ISRO) in undercooled alloy melts. However, "given the local icosahedral coordination of the undercooled liquid, leading to easy formation of icosahedral clusters, it is surprising that these glasses can be cast at low cooling rates," as noted by Xing et al. [29].

In a recent paper by Tanaka [30], a direct correlation between ISRO and the viscosity of the melt has been theoretically analyzed, and the influence of the degree of ISRO in the liquid on its fragility is established. Fragility characterizes the steepness of the viscosity-temperature profile of a melt. In a recent study it was found that Vit1 is a much stronger liquid compared to Vit106 and ZAC $[13,14]$. According to Tanaka's model [30], good glassforming melts, such as Vit1, have a high degree of ISRO over the entire temperature range between their melting and glass-transition temperatures, which contributes to its "strong" liquid behavior. On the other hand, poor glassforming melts (such as pure metals and binary alloys) have a small degree of ISRO near the melting point, which rises significantly with lowering the temperature [31], showing its "fragile" nature.

The greater the degree of polytetrahedral order in the undercooled liquid [i.e., the smaller the value of $\alpha$ in Eq. (5)], the higher its viscosity [32]. The above trend in the $\alpha$ value $\left(\alpha^{\text {Vit1 }}<\alpha^{\text {Vit106 }}<\alpha^{\mathrm{ZAC}}\right.$ ) suggests that the Vit1 melt has the highest degree of polytetrahedral order. The high degree of polytetrahedral order in the case of Vit1 contributes significantly towards its high viscosity and low critical cooling rate for vitrification compared to the other two alloys. Roughly, the time for crystallization scales with the dynamic viscosity of the melt. The small activation barrier promotes prolific nucleation, while high viscosity retards nuclei growth in the case of Vit1. This results in the formation of a large number of small crystals and explains the contradictory experimental finding [22] of nanocrystallization in the case of an exceptional glass former such as Vit1. 
This work was carried out at California Institute of Technology under a contract with the National Aeronautical and Space Administration (Grant No. NNMO4AA21G).

*Corresponding author.

Electronic address: sundeep@caltech.edu

[1] A. Inoue, T. Zhang, and T. Masumoto, Mater. Trans., JIM 31, 177 (1990).

[2] A. Peker and W. L. Johnson, Appl. Phys. Lett. 63, 2342 (1993).

[3] Z. P. Lu, C. T. Liu, J. R. Thompson, and W. D. Porter, Phys. Rev. Lett. 92, 245503 (2004).

[4] C. A. Angell, Science 267, 1924 (1995).

[5] W. L. Johnson, MRS Bull. 10, 42 (1999).

[6] G. Wilde, G. P. Gorler, R. Willnecker, and H. J. Fecht, J. Appl. Phys. 87, 1141 (2000).

[7] J. W. Christian, The Theory of Transformations in Metals and Alloys (Pergamon, Oxford, 2002).

[8] K. F. Kelton, Solid State Phys. 45, 75 (1991).

[9] W. K. Rhim, S. K. Chung, D. Barber, K. F. Man, G. Gutt, A. Rulison, and R. E. Spjut, Rev. Sci. Instrum. 64, 2961 (1993).

[10] S. C. Glade, R. Busch, D. S. Lee, W. L. Johnson, R. K. Wunderlich, and H. J. Fecht, J. Appl. Phys. 87, 7242 (2000).

[11] R. Busch, Y. J. Kim, and W. L. Johnson, J. Appl. Phys. 77, 4039 (1995).

[12] S. Mukherjee, J. Schroers, W. L. Johnson, and W.-K. Rhim (unpublished).

[13] S. Mukherjee, J. Schroers, Z. Zhou, W. L. Johnson, and W.-K. Rhim, Acta Mater. 52, 3689 (2004).
[14] S. Mukherjee, H.-G. Kang, W. L. Johnson, and W.-K. Rhim, Phys. Rev. B 70, 174205 (2004).

[15] D. R. Uhlmann, J. Non-Cryst. Solids 7, 337 (1972).

[16] F. Spaepen, Acta Metall. 23, 729 (1975).

[17] C. V. Thompson, Ph.D. thesis, Harvard University, 1981.

[18] C. V. Thompson and F. Spaepen, Acta Metall. 31, 2021 (1983).

[19] D. M. Herlach, F. Gillessen, T. Volkmann, M. Wollgarten, and K. Urban, Phys. Rev. B 46, 5203 (1992).

[20] D. Holland-Moritz, J. Schroers, D. M. Herlach, B. Grushko, and K. Urban, Acta Mater. 46, 1601 (1998).

[21] W. Kauzmann, Chem. Rev. 43, 219 (1948).

[22] J. Schroers, R. Busch, A. Masuhr, and W. L. Johnson, Appl. Phys. Lett. 74, 2806 (1999).

[23] A. Revesz, P. Donnadieu, J. P. Simon, P. Goyut, and P. Ochin, Philos. Mag. Lett. 81, 767 (2001).

[24] W.-H. Wang, Q. Wei, S. Friedrich, M. P. Macht, N. Wanderka, and H. Wollenberger, Appl. Phys. Lett. 71, 1053 (1997).

[25] H. Assadi and J. Schroers, Acta Mater. 50, 89 (2002).

[26] S. Mukherjee, Z. Zhou, J. Schroers, W. L. Johnson, and W.-K. Rhim, Appl. Phys. Lett. 84, 5010 (2004).

[27] T. Waniuk, J. Schroers, and W. L. Johnson, Phys. Rev. B 67, 184203 (2003).

[28] K.F. Kelton, G. W. Lee, A. K. Gangopadhyay, R. W. Hyers, T. J. Rathz, J. R. Rogers, M.B. Robinson, and D.S. Robinson, Phys. Rev. Lett. 90, 195504 (2003).

[29] L. Q. Xing, Y. T. Shen, and K. F. Kelton, Appl. Phys. Lett. 81, 3371 (2002).

[30] H. Tanaka, J. Phys. Condens. Matter 15, L491 (2003).

[31] T. Schenk, D. Holland-Moritz, V. Simonet, R. Bellissent, and D. M. Herlach, Phys. Rev. Lett. 89, 075507 (2002).

[32] J. D. Bernal, Nature (London) 188, 910 (1960). 